\section{P09.42 SCREENING FOR SYPHILIS, HIV AND HAEMOGLOBIN DURING PREGNANCY IN MOSHI MUNICIPALITY, TANZANIA: HOW IS THE HEALTH SYSTEM PERFORMING?}

${ }^{1} \mathrm{~J}$ Katanga*, ${ }^{2} \mathrm{M}$ Mgongo, ${ }^{2} \mathrm{~T}$ Hashim, ${ }^{3} \mathrm{~B}$ Stray- Pedersen, ${ }^{4} \mathrm{~S}$ Msuya. ${ }^{1}$ Mirembe National Hospital, Dodoma, Tanzania; ${ }^{2}$ Better Health for African Mothers and Children (BHAMC) Tanzania, Moshi, Tanzania; ${ }^{3}$ Div Women and Children, Rikshospitalet, Oslo University Hospital, Institute of Clinical Medicine, University in Oslo, Norway; ${ }^{4}$ Department of Community Medicine, Institute of Public Health, Kilimanjaro Christian Medical University College, Moshi, Tanzania

\subsection{6/sextrans-2015-052270.426}

Introduction Maternal and neonatal morbidity and mortality are still a public health concern in most sub-Saharan Africa (SSA) countries including Tanzania. Among the strategies implemented to reduce maternal mortality rate and neonatal mortality rate and improve outcomes of mothers and newborn babies including universal coverage of quality antenatal care. However the quality of care given to ANC attendees is of great concern in developing countries. This study aimed to provide information on the proportion of pregnant women who were attending for routine ANC at Majengo and Pasua health centres that were tested for $\mathrm{HIV}$, syphilis and $\mathrm{Hb}$.

Methods A cross sectional study was conducted in October 2013 - March 2014. Pregnant women in their $3^{\text {rd }}$ trimester who were attending for routine antenatal care at Pasua and Majengo health centres were enrolled. Interviews were done to determine if women were tested for the 3 tests mentioned in earlier pregnancy, prior to the study followed by clinical examination and blood sample collection to test for HIV, syphilis and $\mathrm{Hb}$. Data were entered and analysed by using SPSS.

Results A total of 536 women were enrolled. Despite being in the third trimester and had attended for routine antenatal care several times, the majority of pregnant women were not screened for syphilis $(89.4 \%),(28.6 \%)$ were not screened for haemoglobin level and only $1 \%$ reported not to be screened for HIV. Three hundred and sixty three participants (87.9\%) reported to have received iron supplement.

Conclusion Syphilis is forgotten and not given the same priority as HIV in pregnant population. Strategies are required to improve its screening as it is the leading cause of stillbirths and perinatal deaths in developing countries like Tanzania

Disclosure of interest statement Authors declare there is no conflict of interest.

\section{P09.43 SEXUALLY TRAMSMITTED INFECTION SCREENING AND FOLLOW-UP IN A HIGH-RISK URBAN OBSTETRIC CLINIC}

1,2S Ona*, 1,2RL Molina, ${ }^{1,2} \mathrm{~K}$ Venkatesh, 1,2K Diouf. 'Department of Obstetrics, Gynecology and Reproductive Sciences, Brigham and Women's Hospital; 'Harvard Medical School, Boston, Massachusetts, USA

10.1136/sextrans-2015-052270.427

Introduction The objective of this study was to determine the screening rate and prevalence of Chlamydia (CT), gonorrhoea (GC), Treponema pallidum (TP), human immunodeficiency virus (HIV) and hepatitis B (HBV) infections among our presumed high-risk obstetric population. We also report treatment and follow-up of patients and their partners.
Methods We conducted a retrospective chart review of 1000 obstetrics patients seen between 2008 and 2014 at our clinic. 854 met criteria for inclusion in the study; included were patients who received most or all prenatal care at our clinic. Demographic data were collected to understand risk factor prevalence in this population. Test results, patients, partner management and follow-up data were extracted from electronic medical records. All descriptive analyses were done using STATA.

Results The mean age of patients was $29.2 \pm 7.5$ (range 14-48). Twenty seven percent reported a prior history of STI, $90 \%$ were screened for CT and GC at least once, 98.9\% for TP, 97.8\% for HBV and $84 \%$ for HIV. Prevalence of CT, GC, CT\&GC, HBV, TP and HIV were $3.7 \%, 0.3 \%, 0.4 \%, 0.2 \%, 0.1 \%$ and $1 \%$, respectively. All patients with positive screening were treated and $97 \%$ of them had a subsequent test of cure. Only $18.2 \%$ of partners were reportedly treated. Thirty nine percent of partners were untreated and management unknown for $42.4 \%$ of partners.

Conclusion Compared to national screening rates, our centre holds very high screening rates but is still not yet optimal. Our HIV screening rate was lowest compared to the other infections. Pregnancy presents a unique opportunity for screening so continuing counselling is indispensable to educate expecting mothers who decline screening on the importance of STIs testing. To address the lack of partners' screening, providing prescription for both patients and their partners should be implemented.

Disclosure of interest statement No funding. No pharmaceutical grants were received in the development of this study.

\section{P09.44 A POCT- ADAPTABLE TEST FOR THE SIMULTANEOUS IDENTIFICATION OF N. GONORRHOEAE AND ITS CIPROFLOXACIN SUSCEPTIBILITY STATUS}

${ }^{1} \mathrm{JR}$ Dillon*, ${ }^{1} \mathrm{~A}$ Taheri, ${ }^{1} \mathrm{NH}$ Khan, ${ }^{1} \mathrm{RP}$ Parti, ${ }^{2} \mathrm{~A}$ Kusalik. ${ }^{1}$ Vaccine and Infectious Diseases Organization, Saskatoon, SK, Canada; ${ }^{2}$ Department of Computer Science, University of Saskatchewan, Saskatoon, Canada

\section{$10.1136 /$ sextrans-2015-052270.428}

Introduction Every year, Neisseria gonorrhoeae (Ng) causes 106 million new gonorrhoea infections worldwide and has recently joined the fast-expanding group of multi-drug resistant superbugs. Treatment is often empirical/delayed as culture-based antibiotic susceptibility tests in current use can take several days. A Point-of-Care Test (POCT) for antimicrobial susceptibility would change this; for example, no longer recommended antibiotics such as ciprofloxacin could be used in certain regions where high percentages of isolates remain susceptible. The objective of the present study was to develop and validate a portfolio of diagnostic primer pairs for application in a Nucleic Acid Amplification-based POCT for the concurrent diagnosis of $\mathrm{Ng}$ and ciprofloxacin susceptibility.

Methods A bioinformatics analysis of $>30,000$ bacterial genomes identified 9 unique signature sequences specific to $\mathrm{Ng}$ FA1090 that were used as targets in a SYBR green-based qPCR assay. 9 diagnostic primer pairs were evaluated for specificity and sensitivity on $271 \mathrm{Ng}$ and non-gonococcal isolates. Two primer pairs targeting ciprofloxacin resistance-conferring single nucleotide polymorphisms in gyrA were tested on 200 resistant and susceptible $\mathrm{Ng}$ isolates. qPCR was performed by an Applied Biosystems StepOnePLus ${ }^{\text {TM }}$ qPCR system. 
Results Out of the 9 diagnostic primer pairs tested for $\mathrm{Ng}$ identification, one probe failed to detect 28 positive samples of $\mathrm{Ng}$ out of a total of 234 isolates. Another primer pair, which amplifies the DR9 repeat region used in the COBAS4800 was not able to detect one $\mathrm{Ng}$ isolate collected from Hong Kong. The remaining 7 primer pairs showed $100 \%$ specificity in terms of $\mathrm{Ng}$ detection and were highly sensitive in detecting $\mathrm{Ng}$ DNA in concentrations as low as $0.00001 \mathrm{ng} / \mathrm{ul}$. A multiplexed assay using ciprofloxacin susceptibility-determining primer pairs distinctly differentiated between resistant and susceptible isolates based on melt curve analysis.

Conclusion A POCT-adaptable assay has been developed for the simultaneous identification of N. gonorrhoeae and its ciprofloxacin susceptibility status.

Disclosure of interest statement The present work was supported by Grand Challenges Canada ((\#S5 398). No grants were received from any company in the development of this study.

\section{P10 - Human papillomavirus infections and other viral STI}

\section{P10.01 HIGH-RISK HPV IS A MARKER FOR ATYPICAL INTRAANAL CYTOLOGY IN IMMUNOCOMPETENT WOMEN}

${ }^{1}$ E Eleutério, ${ }^{2}$ MRL Passos ${ }^{*},{ }^{1} \mathrm{R}$ Eleutério, ${ }^{3} \mathrm{P}$ Giraldo, ${ }^{4}$ AK Gonçalves. ${ }^{1}$ Universidade Federal Do Ceará, Fortaleza, Brazili, ${ }^{2}$ STD Clinic, Universidade Federal Fluminense, Niterói, Brazili; ${ }^{3}$ Universidade Estadual de Campinas, Campinas, Brazil; ${ }^{4}$ Universidade Federal Do Rio Grande Do Norte, Natal, Brazil

\subsection{6/sextrans-2015-052270.429}

Introduction Currently there is a worldwide concern about how best to screen for anal intraepithelial lesion. The cytology seems to be a good method, but there are few experts who have skill. The objective of this study was to evaluate whether high-risk HPV identification by PCR may precede cytology this end.

Methods This was an cross-sectional study of 140 women attended at the Federal University of Ceará (UFC). A sample of the residual material of liquid-based cytology (Surepath ${ }^{\circledR}$ ) was used for RT-PCR on the Cobas 4800 (Roche). Cytological findings were compared with the PCR results. Fisher exact test were applied for a CI of $95 \%$.

Results There were 57/140 positives cases for high-risk HPV and $83 / 140$ negative cases. The average age was similar in both groups. The number of sexual partners referred was not significantly different between the two groups. Among the positive cases atypical intra-anal cytology was significantly more frequent. Among the HPV positive $=17(25 \%)$ and in negative $=12$ $(14.5 \%)(\mathrm{RR}=2.06,95 \% \mathrm{CI}=1.06$ to 3.98$)$.

Conclusion The presence of intraanal high-risk HPV is frequent and its presence is associated with an increased risk of abnormal intraanal cytology.

Disclosure of interest statement There is no conflict of interest.
P10.02 FIELD EVALUATION OF THE XPERT HPV TEST FOR THE DETECTION OF HUMAN PAPILLOMAVIRUS INFECTION IN WOMEN USING SELF-COLLECTED VAGINAL COMPARED TO CLINICIAN-COLLECTED CERVICAL SPECIMENS

${ }^{1,2}$ A Vallely*, ${ }^{2} \mathrm{P}$ Toliman, ${ }^{3} \mathrm{~S}$ Tabrizi, ${ }^{1} \mathrm{SG}$ Badman, ${ }^{4} \mathrm{Z}$ Kombati, ${ }^{2} \mathrm{~J}$ Gabuzzi, ${ }^{2} \mathrm{~J}$ Allan, ${ }^{2} \mathrm{G}$ Munnull, ${ }^{2} \mathrm{~S}$ Silim, ${ }^{5} \mathrm{C}$ Ryan, ${ }^{2} \mathrm{LM}$ Vallely, ${ }^{2,6} \mathrm{~A}$ Kelly-Hanku, ${ }^{1} \mathrm{H}$ Wand, ${ }^{7} \mathrm{GDL}$ Mola, ${ }^{2} \mathrm{P}$ Siba, ${ }^{1} \mathrm{R}$ Guy, ${ }^{1} \mathrm{JM}$ Kaldor. ${ }^{1}$ The Kirby Institute, University of New South Wales, Sydney, Australia; ${ }^{2}$ Papua New Guinea Institute of Medical Research, Goroka, Papua New Guinea; ${ }^{3}$ Molecular Microbiology Laboratory, The Royal Women's Hospital, Melbourne, Australia; ${ }^{4}$ Pathology Department, Mt Hagen Hospital, Western Highlands Province, Papua New Guinea; ${ }^{5}$ The Burnet Institute, Melbourne, Australia; ${ }^{6}$ School of Public Health \& Community Medicine, UNSW Australia; ${ }^{7}$ Department of Obstetrics \& Gynaecology, School of Medicine and Health Sciences, University of Papua New Guinea, National Capital District, Papua New Guinea

\subsection{6/sextrans-2015-052270.430}

Background The Cepheid Xpert ${ }^{\circledR}$ HPV Test has high sensitivity and specificity for the detection of HPV infection in women compared to the Roche cobas $4800 \mathrm{HPV}$ assay using cervical specimens. In many high-burden, low-resource countries it will not be feasible to achieve high cervical screening coverage using HPV-DNA technologies that require clinician-collected samples. We are conducting the first evaluation of self-collected vaginal specimens compared to clinician-collected cervical specimens for the detection of HPV infection using the Xpert ${ }^{\circledR}$ HPV Test. This study is being conducted in Papua New Guinea, which has among the highest rates of cervical cancer globally, with an agestandardised incidence of $23.7 / 100,000$ compared to 5.0/ 100,000 in Australia and New Zealand.

Methods Women aged 30-54 years attending two Well Woman Clinics are invited to participate and following informed consent procedures, complete a short interview, clinical examination, and provide self-collected and clinician-collected cytobrush specimens for clinic-based HPV testing. Women are given their cervical test result the same day. Those with a positive HPV test and a positive examination on visual inspection of the cervix with acetic acid are offered same-day cervical cryotherapy.

Results A total of 313 women were recruited to end-Feb 2015. There was $94.2 \%$ overall percentage agreement (OPA) between vaginal and cervical tests for all high-risk HPV (hrHPV) types; 100\% OPA for HPV-16; and 99.7\% OPA for HPV 18/45. Based on cervical test results, the prevalence of HPV-16 was $4.2 \%(13 /$ 313); HPV $18 / 45$ was $1.6 \%$ (5/313); and other hrHPV, $11.8 \%$ (37/313). Overall, 15.7\% (49/313) of participants had one or more hrHPV infection.

Conclusion Preliminary results suggest that self-collected vaginal specimens compare favourably to clinician-collected cervical specimens for the detection of HPV infection using the Xpert ${ }^{\circledR}$ HPV Test. If confirmed, this finding is likely to have significant implications for future HPV-based cervical screening programs in high-burden, low-resource settings worldwide.

Disclosure of interest statement Nothing to Disclose.

\section{P10.03 ANAL HUMAN PAPILLOMAVIRUS (HPV) INFECTION AND ANAL INTRAEPITHELIAL NEOPLASIA (AIN) AMONG MEN WHO HAVE SEX WITH MEN (MSM) IN KUALA LUMPUR, MALAYSIA}

I Azwa*, I Yee, N Hairunisa, CH Tan, S Harun. Clinical Investigation Centre, University Malaya Medical Centre, Kuala Lumpur

10.1136/sextrans-2015-052270.431 